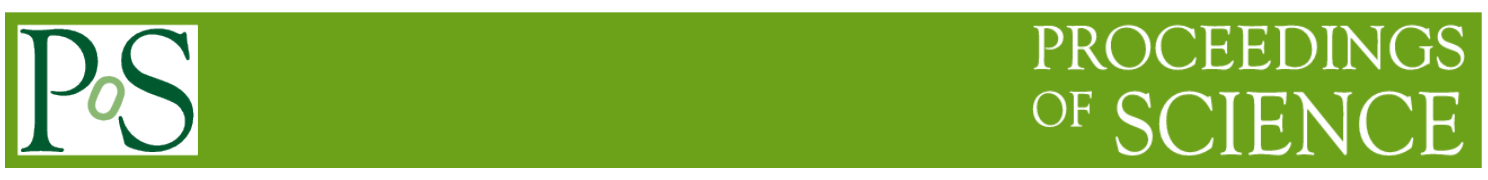

\title{
CHARM Observatory Status and performance
}

\author{
Cotzomi J. ${ }^{1}$, Conde R., Salazar H., Matinez O., Hernández E., Ponce E. \\ Benemérita Universidad Autónoma de Puebla \\ Av. San Claudio y 18 sur, colonia San Manuel Puebla México. \\ E-mail:jcotzomi@yahoo.com.mx
}

CHARM, Cosmic High Altitude Radiation Monitor, is an observatory whose target is the study of cosmic rays. This is actually under construction stage, it consists of an array of Water Cherenkov Detectors (WCD) located in Puebla México, at 18.591N and 97.1876W, 4300 meters over sea level. Each WCD container inner walls is covered inside with a high UV reflective material called Tyvek, and it is filled with 12000 liters of pure water. The WCD has a sensitive area of $10 \mathrm{~m}^{2}$, and the Photomultiplier inside (PMT) is an EMI 9530A (PMT), which has the acquisition electronic associated too. The observatory is designed to study high-energy cosmic rays in the range $1 \mathrm{PeV}-$ $100 \mathrm{PeV}$. This work presents the actual status, the temporal structure signals (peak amplitude and integrated charge) and the stability of the Water Cherenkov Detectors. This study is performed using secondary particles from the background.

35th International Cosmic Ray Conference - ICRC2017

10-20 July, 2017

Bexco, Busan, Korea

${ }^{1}$ Speaker 


\section{Introduction}

The unique method for the study of high energy cosmic rays is through Extensive Air Showers (EAS). According to experimental data [1] [2], EAS at energies above $3 \mathrm{PeV}$ present a decreased flow of protons and an increase in heavy nuclei of primary cosmic rays. This change in the count rate reaches the heavy component of cosmic rays with energies above $500 \mathrm{PeV}$, decreasing the flow of heavy nuclei. What it is expected is that to energies higher than $100 \mathrm{PeV}$, cosmic rays from other sources such as extragalactic, should contribute the most on primary cosmic rays flow.

Studies carried by KASCADE array [3] obtained a change of steepening in the energy spectrum of cosmic rays about $5 \mathrm{PeV}$ for the all-particles, however the measurements of Tunka Array[1] established the change steepening at about $3 \mathrm{PeV}$. The steepening change called "Knee" was established for the KASCAGE-Grande Collaboration [4] for the heavy component of cosmic rays about $80 \mathrm{PeV}$, so it is not yet clear what causes and the value of the breaks in the experimental energy spectrum of cosmic rays.

The CHARM observatory is an array of high altitude under construction located at an approximate depth of $630 \mathrm{~g} / \mathrm{cm} 2$ (4300m.a.s.l.). CHARM has the advantage of observing the maximum or the beginning of development (depending of energy $\mathrm{CR}$ ) of the secondary particles of EAS generated by primary cosmic rays. One goal of CHARM is to contribute to the study of slope change of energy spectrum and mass composition of the primary particles of the cosmic rays with energy between $1 \mathrm{PeV}$ to $100 \mathrm{PeV}$, using the capabilities and advantages of its location.

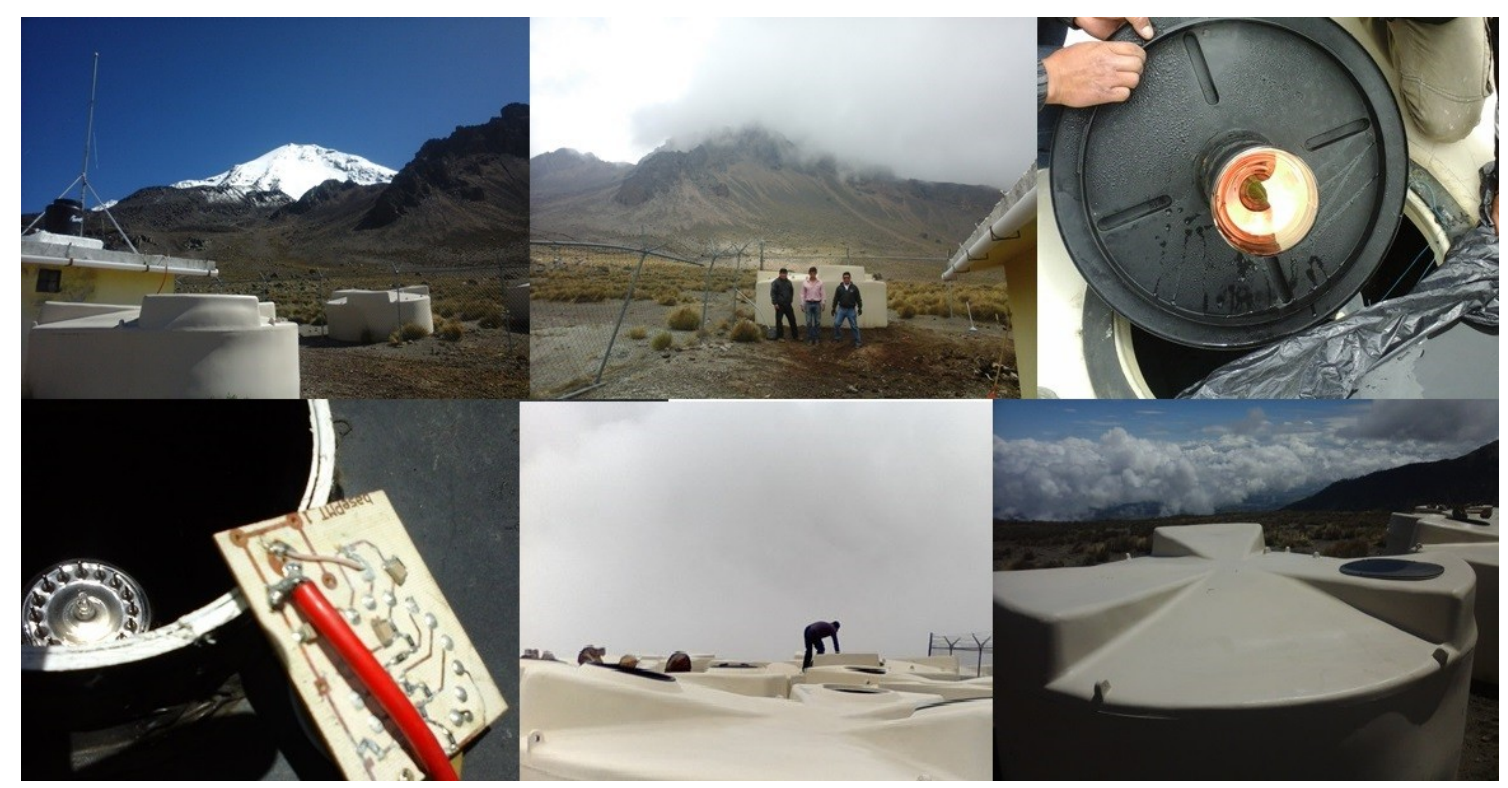

Fig.1 The CHARM Observatory site, located near at Pico de Orizaba Volcano at 4300m.a.s.1. In these pictures can see the tanks, eolic system of generating energy, the PMTs and electronic associated. 


\section{The CHARM Observatory}

\subsection{The experimental array}

CHARM Observatory is an array of Water Cherenkov Detectors (WCD) located at 4300 m.a.s.1., at $18.5 \mathrm{~N}$ and $97.18 \mathrm{~W}$. CHARM is a high altitude observatory designed to study highenergy cosmic rays in the range of $10^{15}-10^{17} \mathrm{eV}$ of energy, that consists of 25 light-tight cylindrical containers of $10 \mathrm{~m}^{2}$ cross section. It filled with 12000 liters of purified water, covered in the inner walls with Tyvek, material of high reflectivity, see figure No. 1. The detectors are distributed in 3 hexagonal rings. In the first stage we considerate three tanks of the array in order the stability monitoring an evaluating performance of the detectors. Each station consist in a Photomultiplier (PMT) EMI 9530A with a 5" sensitive area. This PMT is located on the top looking downwards, including the Data Acquisitions System (DAQ) non commercial and power supply electronics.
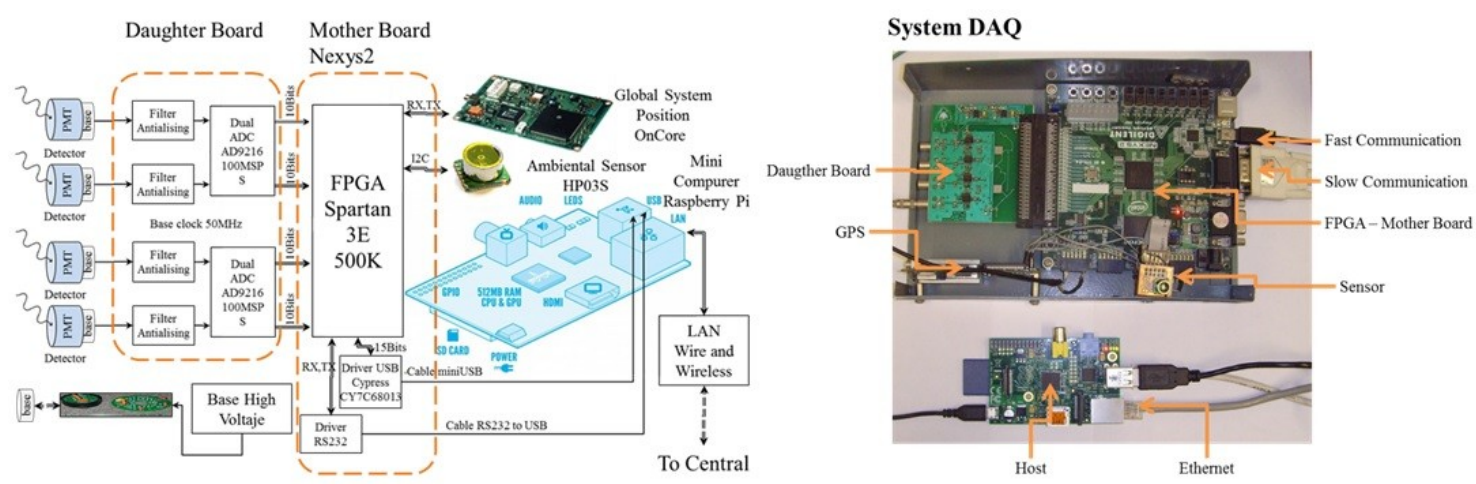

Fig.2 The acquisition electronic data system of the CHARM Observatory.

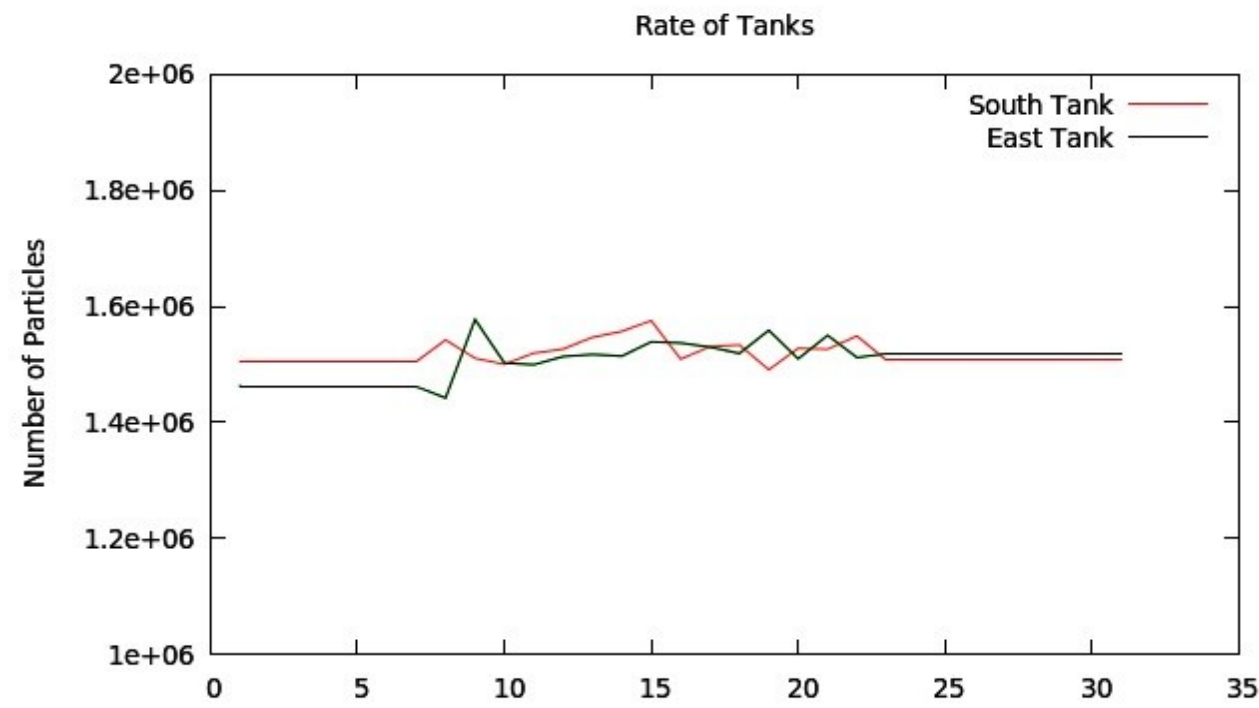

Fig.3 Rate of two tanks for the stability monitoring of detectors. 


\subsection{The DAQ System}

In general, the associated electronic for the acquisitions data of array is based on a fourchannel Analog Digital Converter (ADC) Daughter Board with a rate of 100MSPS, PMTs, a Field Programmable Gate Array (FPGA) Mother Board, and a mini computer Raspberry PI [5].

The DAQ system includes a 4-channel ADC Daughter Board with two dual 10-bit ADC chips from Analog Devices. Each chip was the AD9216 that is a dual, 3V, 10-bit, 105 Mega Samples Per Second (MSPS) to converted analog to digital. The Daughter Board is connected to a second board with an FPGA Nexis2 for the data processing. The communication and control are based on a small, Raspberry PI model B 512MB minicomputer, that features an ARM1176JZF-S $700 \mathrm{MHz}$ processor. This minicomputer is the main control between the host and the DAQ, and it is used for interconnection with the surface detector modules; the Mother Board has two ways of communication with the host, the first use a serial port with a typical communication rate around 115200 bits per second and the second one uses a USB port with a higher communication speed. (See figure No. 2)
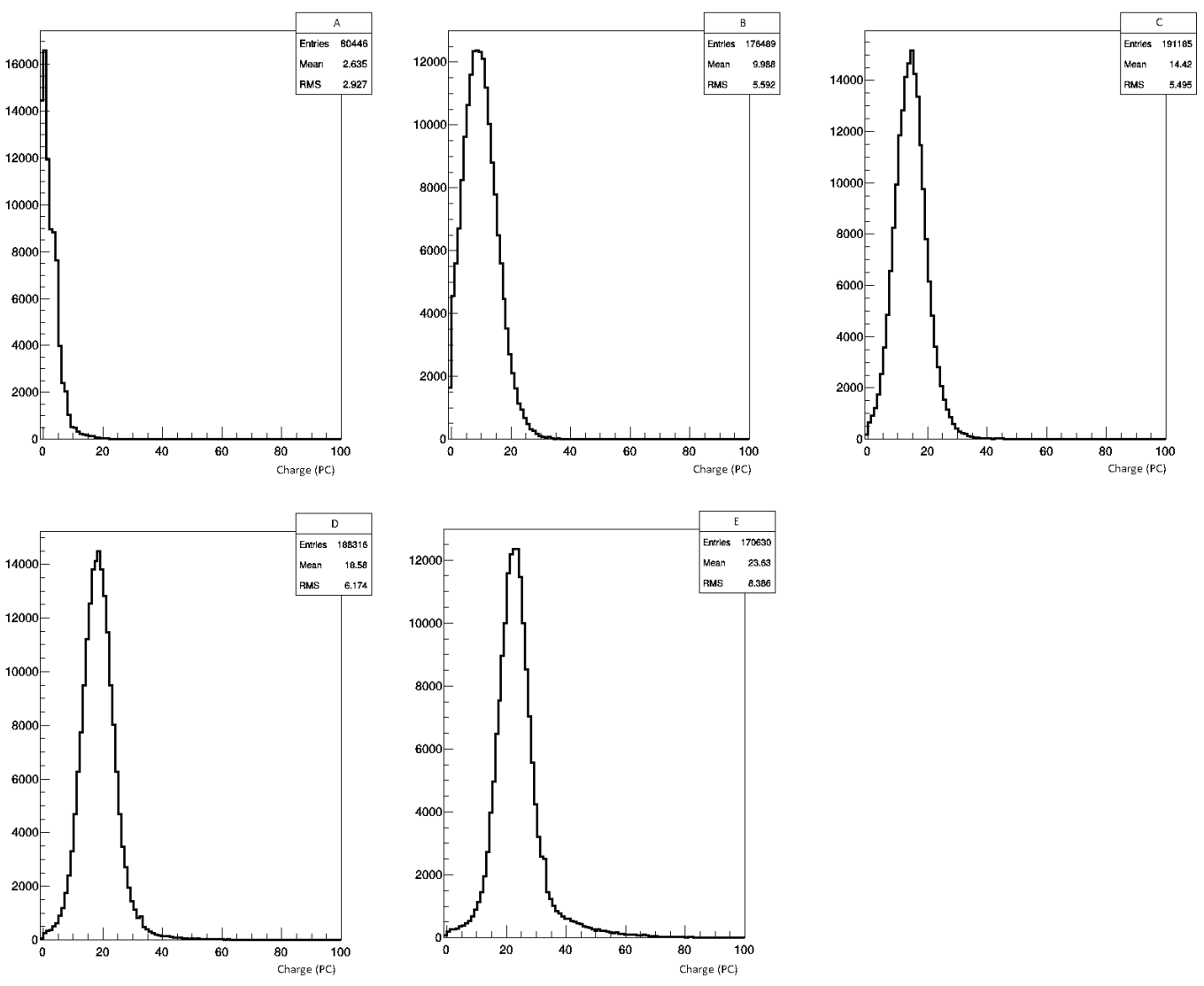

Fig.4: Histograms of Integrated Charge for trigger level equals to, histogram A:2mV, B:4mV, $\mathrm{C}: 6 \mathrm{mV}, \mathrm{D}: 8 \mathrm{mV}$ and $\mathrm{E}: 10 \mathrm{mV}$. 


\subsection{Acquisitions data}

We registered in the WCD secondary particles of the background radiation of the cosmic rays acquired form the associated electronic in form of pulse. The pulse profile corresponds to the increasing part of the electronic signal, It consists of 16 consecutive samples (the digitalization is performed every $10 \mathrm{~ns}$, so a trace of 16 samples corresponds to $160 \mathrm{~ns}$ ). The condition to capture a trace of event requires that the signal exceed the trigger level 2, 4, 6, 8, and $10 \mathrm{mV}$. These samples are separated in two groups, the first 7 samples are part of trace before passing the trigger level, the last 9 samples are the pulse profile and corresponds to the samples with exceed the trigger level, with this we can take an analysis of the acquired pulses. Of the above, the average of first 3 samples determinate the base line and the remaining 13 samples are integrated for the determinate of the integrated charged, this is make for the each pulse acquired.

\section{Results and discussions}

This study is performed using the secondary particles of the background radiation of the cosmic rays. In this work we present temporal structure studies and the rate of acquired signals of three WCD of CHARM. We measured the rise time parameter, peak amplitude and integrated charge of the signals obtained from each detector.

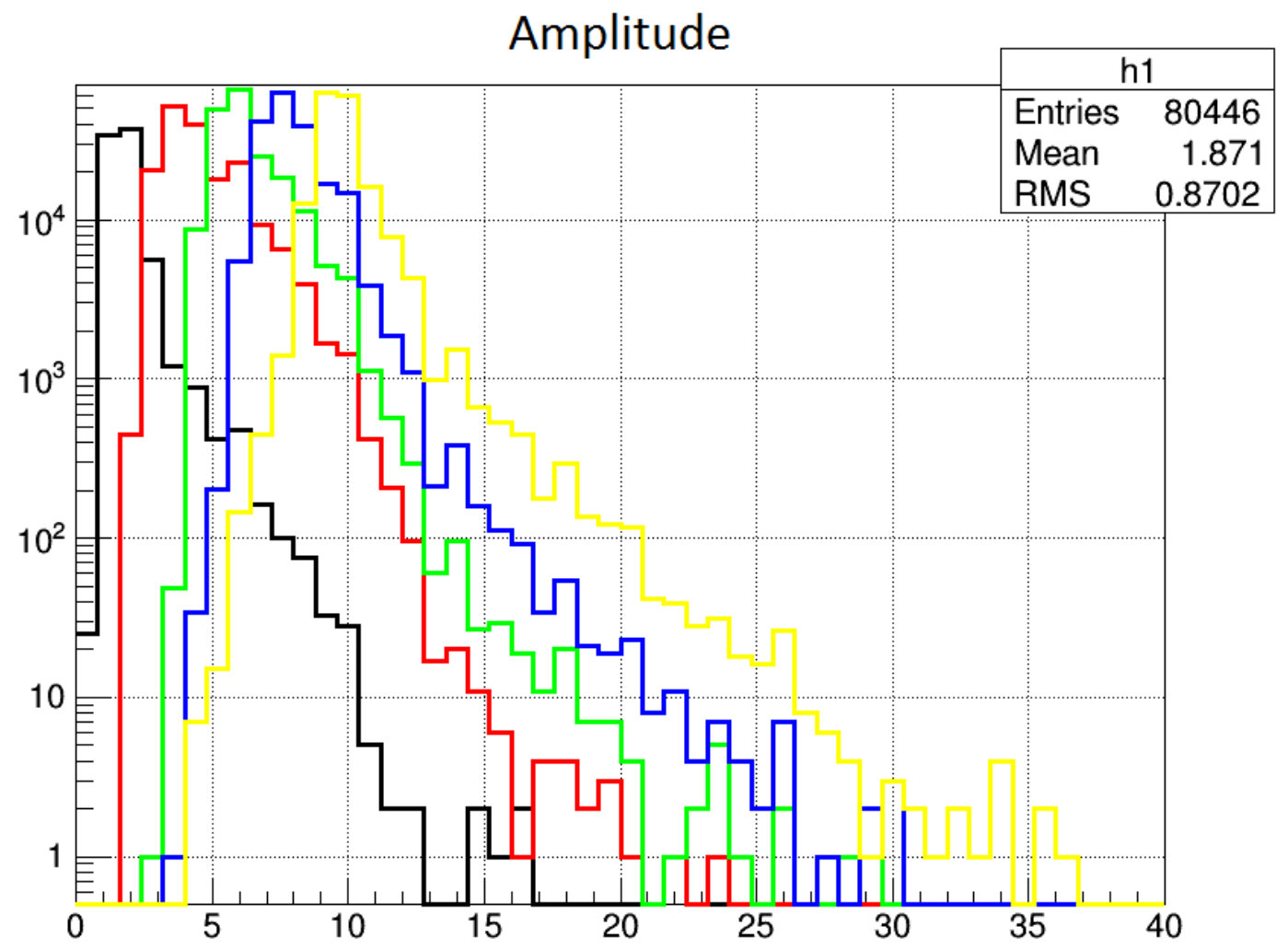

Fig.5 Histograms of Amplitude of signals of a trigger level of black: $2 \mathrm{mV}$, red: $4 \mathrm{mV}$, green: $6 \mathrm{mV}$, blue: $8 \mathrm{mV}$ and yellow: $10 \mathrm{mV}$. 
A method based mainly on single particle rates was used as a simple estimator of the stability of detectors. The condition for the count rate is that the signal acquired exceed the trigger levels $(2,4,6,8,10 \mathrm{mV})$, obtaining high count rate for low trigger level that the high trigger levels, see the figure 3 .

We can selected the Amplitude of signals from the WCD with the thresold level associated to electronic system DAQ. We can operated the WCD with a low threshold level (2, 4 , o $6 \mathrm{mV}$ ) to detect single electrons and muons from the background radiation of the cosmic rays, it from the signals measurement in each detector into the number of particles from the EAS reaching the detector. Each signal detected has one temporal profile associated to the nature of the particle, this structure is rich information due to their wide spread in arrival time. So, from the signals of the WCD it is possible to obtain information about ratio of muons to electromagnetic components. The study of this ratio is very important because this parameter is relationated with mass compositions of the primary cosmic ray. According to acquired signals from WCDs, we show statisticaly mean signal detected of the WCD of CHARM to the identification of the electrons and muons signals observed in the histograms of Integrated Charge and Amplitude or Voltage of each signals detected, see figures 4 and 5 respectively.

Each curve corresponds to a trigger level: the histogram A corresponds to a trigger level equal to $2 \mathrm{mV}, \mathrm{B}$ is $4 \mathrm{mV}, \mathrm{C} 6 \mathrm{mV}$, D is $8 \mathrm{mV}$, and $\mathrm{E}$ is equal to $10 \mathrm{mV}$. For the triggers levels low we expect a contribution of more individuals particles as electrons and muons, however for triggers levels high, the charge is generated by showers that hit the WCD. This same correlation can see in the figure 5. For example, for the histogram H5 with trigger level of $10 \mathrm{mV}$, can see acumulations events with amplitudes greater than $10 \mathrm{mV}$. The mean of each histogram increases as increases the trigger level value.

\section{T50 vs T90}

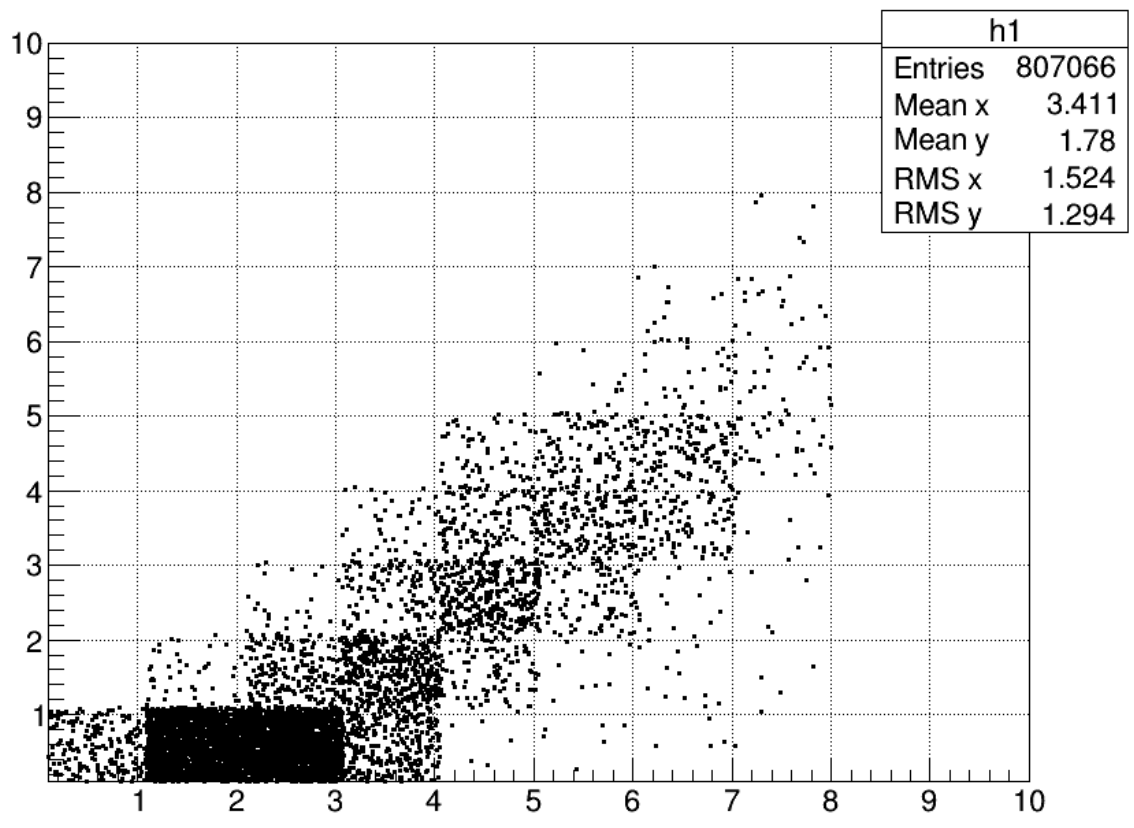

Fig.6 The correlation between times T50 and T90\%. 
As we indicated above, the rise time is a parameter rich of information about the nature of particles, we determinate pulses signals widths, T50 and T90. T50 is the time interval in wich the integrated charged of signals rises from $10 \%$ to $50 \%$, and T90\% is the time interval from $10 \%$ to $90 \%$, see figure 6 . More complex monitoring can be done by using bi-dimensional correlation plots, Charge vs. risetime 10-90\%, as shown in figure 7; this time is when reaching a value equal to $90 \%$ of the integrated charge.

The plots shown in the figure 7, can be used to separate many of the physical processes that occur inside WCD. The events in the plot h4 were registered with a trigger level of $4 \mathrm{mV}$, it is visible an accumulation of events in the region $6<$ charge $<20 \mathrm{pC}$ and rise $2<$ times $<10 \mathrm{~ns}$ that corresponds mainly to events of isolated electrons. In this region is clearly visible the acumulation in the number of events around $5 \mathrm{~ns}$; at rise time $>10 \mathrm{~ns}$ and charge $>11 \mathrm{pC}$ that correspond to single muons mainly in a low number. The registered events in Plot h6 were taken with a trigger level of $6 \mathrm{mV}$, and it is visible an accumulation of events in the region $10<$ charge $<30 \mathrm{pC}$ and $3<$ rise times $<15 \mathrm{~ns}$ that corresponds to individuals muons and menor contribution the electromagnetic particles generated ot decay of muons into the WCD. This contribution is present at rise time $>10 \mathrm{~ns}$ and charge $>20 \mathrm{pC}$ for the trigger level of $10 \mathrm{mV}$, and in these regions there are also single muons in a low number, as the events at the end of the graph correspond to small showers registered by the WCD. In all cases, this corresponds to the mean charge deposited by particles crossing the detector in arbitrary directions
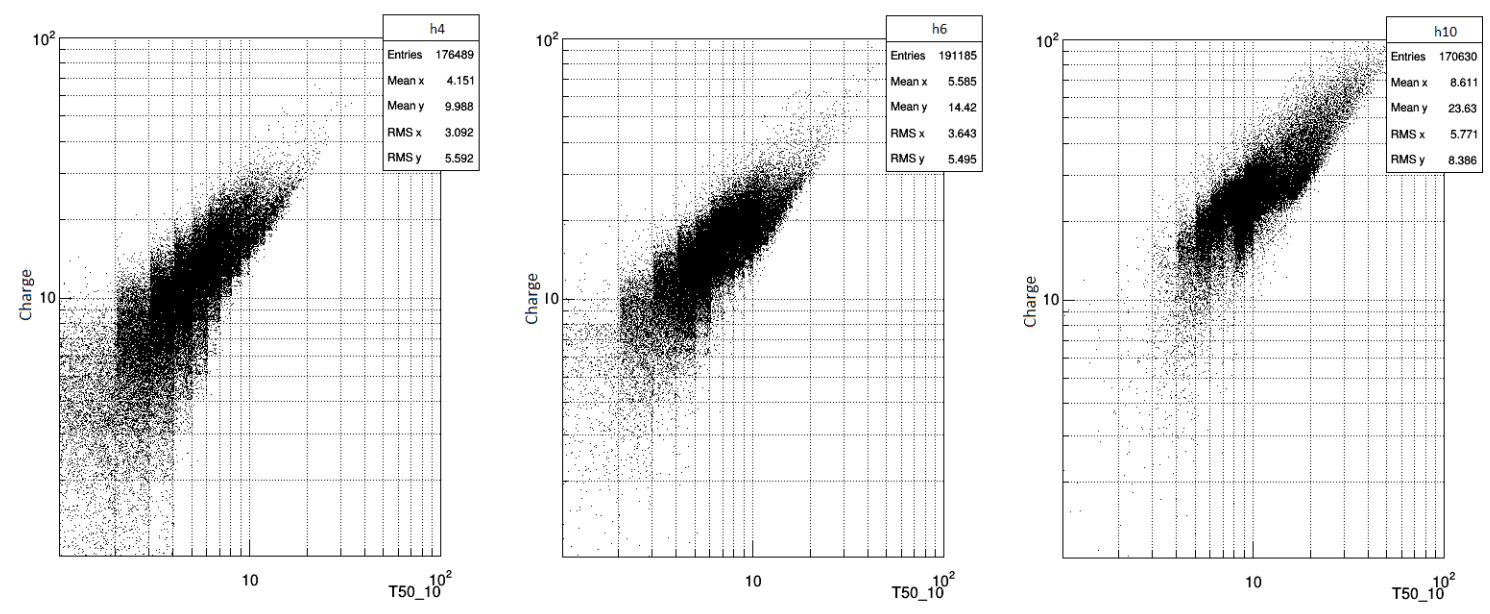

Fig.7 The graphs correspond to events of integrated charged vs difference time T10-T90. h4, h6 and h10 are the graphs for events with trigger levels of 4, 6 and $10 \mathrm{mV}$ respectively.

\section{Conclusions}

In this work we report the results of the rate events, the temporal structure, amplitude and integrated charge of signals of detectors WCD of CHARM at 4300 m.a.s.l. With this we test the stability of detectors, DAQ system, the electronic asosciated, the response and adquisitions of signals of WCDs of HAWC. As seen in the graphs, it is possible see the work 
cycle in the detectors. According to level triggers it was possible to register individual electrons and muons, electromagnetic particles generated by shower into tanks. We show that the amplitude of the signals produced into the Water Cherenkov Detectors are proportional to their geometric path lengths as see in the graphs.

\section{Acknowledgements}

The authors are gratefully acknowledge for the continuous financial support from the VIEP BUAP.

\section{References}

[1] N. Budnev, et. al., Tunka-25 Air Shower Cherenkov array: The main results, Astroparticle Physics, 50-52 (2013), 18-25.

[2] T. Antoni, et. al., The cosmic-ray experiment KASCADE, NIM A, 513 (2003) 490-510.

[3] T. Antoni, et. al., KASCADE measurements of energy spectra for elemental groups of cosmic rays. Results and open problems. Astroparticles Phys. 24 (2005) 1.

[4] W. D. Apel., et. al., KASCADE Grande Collaboration, The spectrum of Cosmic rays measurement with KASCADE Grande, Astroparticle Phys. 36 (2012) 183.

[5] Conde R., et. al., Desing and implementation of a data acquisition system for an array of cosmic rays detectors, IJAREEIE, Vol.2, Issue 10, (2013). 